\title{
Intraoperative Diagnosis and Surgical Procedure with Imprint Cytology for Small Pulmonary Adenocarcinoma
}

\author{
Tomoyuki Nakagiri ${ }^{1}$; Tomio Nakayama ${ }^{2}$; Toshiteru Tokunaga ${ }^{1}$; Akemi Takenaka ${ }^{3}$; Hidenori Kunoh ${ }^{1}$; \\ Hiroto Ishida ${ }^{1}$; Yasuhiko Tomita ${ }^{4}$; Shin-ichi Nakatsuka ${ }^{4}$; Harumi Nakamura ${ }^{4}$; Jiro Okami ${ }^{1}$; Masahiko \\ Higashiyama ${ }^{\circledR}$
}

1. Department of General Thoracic Surgery, Osaka International Cancer Institute, Osaka, Japan

2. Cancer Control and Statistics, Osaka International Cancer Institute Osaka, Japan

3. Department of Cytology, Osaka International Cancer Institute, Osaka, Japan

4. Department of Pathology, Osaka International Cancer Institute, Osaka, Japan

$\square$ Corresponding author: Masahiko Higashiyama, MD, PhD, Department of Thoracic Surgery, Osaka Medical Center for Cancer and Cardiovascular Disease, Osaka, Japan. 1-3-3 Nakamichi, Higashinari-ku, Osaka 537-8511, Japan. Tel: +81 66972 1181; Fax: +81 669818055

(c) The author(s). This is an open access article distributed under the terms of the Creative Commons Attribution License (https://creativecommons.org/licenses/by/4.0/). See http://ivyspring.com/terms for full terms and conditions.

Received: 2019.03.19; Accepted: 2020.01.22; Published: 2020.02.20

\begin{abstract}
Objectives: For patients with multiple small-sized pulmonary cancers, a lobectomy can disrupt future therapeutic options for other lesions. It was recently reported that limited pulmonary resections were not inferior to lobectomy for the management of selected peripheral small-sized pulmonary adenocarcinomas. Patients with adenocarcinoma in situ or minimally invasive adenocarcinoma, as proposed by the International Association for the Study of Cancer classification, have been reported to have $100 \%$ survival after 5 years. However, that classification can be applied postoperatively. Since 2005 , we have been intentionally performing limited pulmonary resection procedures for small-sized adenocarcinoma cases based on intraoperative imprint cytological diagnosis and our classification (Nakayama-Higashiyama's classification).

Materials and Methods: A total of 120 consecutive cases were included in this study. Lung tumors were removed intraoperatively by wedge resection, and stump smear cytology was performed, from which the cases were classified into 5 groups based on our classification. When the tumor was classified as Group I or II, the operation was finished. When diagnosed as a more advanced classification, a lobectomy and lymph node dissection were additionally performed.

Results: The 5-year survival rate for Group I and II was 100\%, while those for Group III and IV-V were $95.8 \%$ and $94.4 \%$, respectively. The 5-year disease-free survival rates for Group I and Group II were $100 \%$ and $97.1 \%$, respectively, and for Group III and IV-V they were $100 \%$ and $94.1 \%$, respectively.

Conclusion: Use of cytological findings along with Nakayama-Higashiyama's classification for determining operation procedure is effective for treatment of patients with small-sized pulmonary adenocarcinoma.
\end{abstract}

Key words: small size lung adenocarcinoma, intraoperative diagnosis, surgical procedure, outcome, Nakayama-Higashiyama's classification

\section{Introduction}

With the improved resolution of computer tomography (CT) and/or spread of CT screening, we often find small multiple ground-glass opacities (GGOs) in lesions in the lungs. It is often difficult to diagnose these lesions with bronchoscopy alone. Intraoperative diagnosis is often needed for these lesions. In addition, the incidence of second primary lung cancer after resection of non-small cell lung cancer (NSCLC) has been estimated to be $1 \%$ to $4 \%$ per patient-year, ${ }^{1-4}$ and even after resection of stage I NSCLC, the incidence was reported to be $2 \%$ per patient-year. ${ }^{4}$ In such cases, a lobectomy can disturb future therapeutic options for other lesions or second primary lesions.

It was recently reported that a limited pulmonary resection procedure, including a wedge resection and segmentectomy, was not inferior to lobectomy for the management of peripheral 
small-sized pulmonary adenocarcinomas, especially adenocarcinoma in situ (AIS) or minimally invasive adenocarcinoma (MIA) in the International Association for the Study of Cancer (IASLC)/American Thoracic Society (ATS)/European Respiratory Society (ERS) classification of lung adenocarcinoma. ${ }^{5,6}$ The survival of patients with AIS or MIA is $100 \%$ at 5 years after the operation. ${ }^{7}$ In the early stage of such types of lung cancers, it is better to resect the lesion with wedge resection, which needs limited hilar vessel handling compared to segmentectomy, considering the potential need for future surgery. To decide whether to perform a limited pulmonary resection or lobectomy, a method of intraoperative diagnosis is needed.

For conventional intraoperative diagnosis, a biopsy, especially partial resection of the tumor, is needed, despite the small size of the tumor. However, according to the classification of the IASLC $8^{\text {th }}$ TNM system, we have to describe the tumor by size, invasion of peripheral structures, and as adenocarcinoma, type of adenocarcinoma subclassification as the postoperative diagnosis. With the conventional intraoperative biopsy (or partial resection) for cryosection, part of the tumor is lacking. As a result, one cannot accurately diagnose the tumor pathologically after the operation. To diagnose the tumor accurately, we have to conserve the tumor, as much as possible; thus, another intraoperative diagnosis method is needed.

Previously, we reported a cytological method, termed Nakayama-Higashiyama's classification $(\mathrm{N}-\mathrm{H}$ classification), in which imprint cytology findings of small adenocarcinomas are correlated with the IASLC adenocarcinoma pattern classification, WHO $4^{\text {th }}$ adenocarcinoma histological grading, and the $8^{\text {th }}$ IASLC TNM classification. ${ }^{8}$ Although other studies have also reported the usefulness of cytological smear findings for small-sized pulmonary adenocarcinoma cases,, 10 there are no reports of its clinical application. Since August 2005, we have been intentionally performing limited pulmonary resection procedures for small-sized adenocarcinoma cases based on intraoperative imprint cytological diagnosis and the $\mathrm{N}-\mathrm{H}$ classification. The results of a comparison between the classification and GGO rates, and a prospective study of our procedure selection are presented.

\section{Materials and Methods}

\section{Sample collection}

The lung tumors in the present patients were resected by wedge resection. The samples were cut at the center of the tumor and smeared onto microscope slides. The preparations were immediately fixed in the operation room and evaluated (Supplement Fig. $\left.1^{8}\right)$.

\section{Classification}

Since 1993, we have been performing imprint cytology intraoperatively and classifying the samples into 5 groups based on the N-H classification (Table 1, Supplement Fig. $2^{8}$ ), as recently reported. ${ }^{8}$ For the present study, adenocarcinomas smaller than $2 \mathrm{~cm}$, excluding mucinous adenocarcinoma cases, were examined.

\section{Patients}

Since August 2005, we have been performing limited surgery with intraoperative imprint cytology based on the N-H classification. The present study included consecutive patients with small lung adenocarcinoma $(\leq 1.5 \mathrm{~cm})$ or GGO-predominant small adenocarcinoma $(\leq 2.0 \mathrm{~cm})$ tumors. Patients with clinical T1a-bN0 lung adenocarcinomas according to the $8^{\text {th }}$ edition and who underwent surgery from August 2005 to February 2010 and subsequent follow-up were analyzed. Written, informed consent was obtained from all patients, and the ethical review board of our institution approved this study (Approval No. 18055).

Table 1. Nakayama-Higashiyama's classification of small pulmonary adenocarcinoma ${ }^{8}$

\begin{tabular}{|c|c|c|c|c|c|}
\hline & Group I & Group II & Group III & Group IV & Group V \\
\hline Cellularity & poor & moderate & hyper & hyper & hyper \\
\hline Size of cluster & $10-30$ cells & slightly large cluster & small to large cluster & single to large cluster & single to large cluster \\
\hline Shape of cluster & sheet-like appearance & $\begin{array}{l}\text { mainly sheet-like appearance, } \\
\text { partly overlapping }\end{array}$ & irregular overlapping & $\begin{array}{l}\text { scattered isolated cells to } \\
\text { irregular overlapping }\end{array}$ & $\begin{array}{l}\text { scattered isolated cells to } \\
\text { irregular overlapping }\end{array}$ \\
\hline Size of cells & small & small to medium & small to large & large & large \\
\hline Dyskaryosis & none & slight & often & often & marked \\
\hline Size of nucleus & small \& uniform size & $\begin{array}{l}\text { small to medium \& } \\
\text { anisokaryosis }\end{array}$ & $\begin{array}{l}\text { small to large \& } \\
\text { anisokaryosis }\end{array}$ & large \& anisokaryosis & large \& anisokaryosis \\
\hline Chromatin pattern & $\begin{array}{l}\text { thick, fine and granular } \\
\text { chromatin with regular } \\
\text { distribution }\end{array}$ & $\begin{array}{l}\text { thick to sparse and fine, } \\
\text { granular chromatin }\end{array}$ & $\begin{array}{l}\text { fine granular chromatin with } \\
\text { irregular distribution }\end{array}$ & $\begin{array}{l}\text { fine granular chromatin with } \\
\text { irregular distribution }\end{array}$ & $\begin{array}{l}\text { fine to coarse, granular } \\
\text { chromatin with irregular } \\
\text { distribution }\end{array}$ \\
\hline $\begin{array}{l}\text { Distance of } \\
\text { inter-nucleus }\end{array}$ & slightly irregular & slightly irregular & irregular & irregular & irregular \\
\hline
\end{tabular}


GGO rate $(\%)$

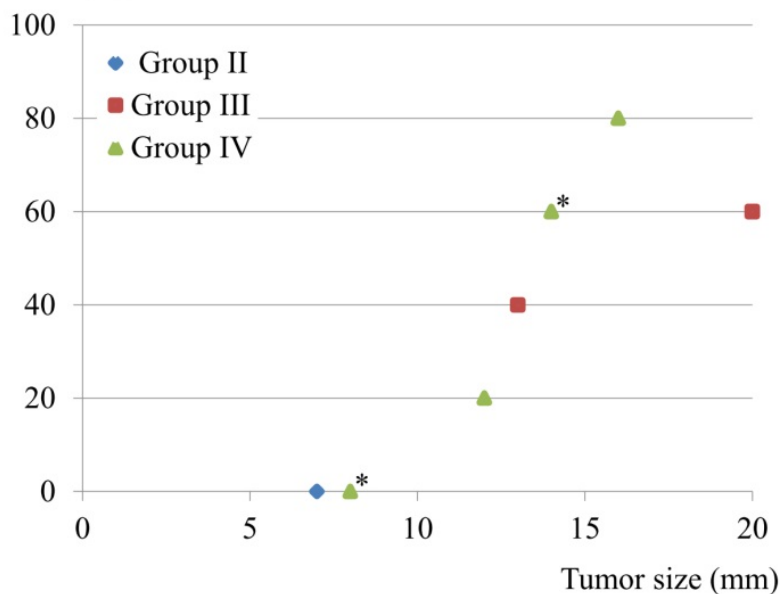

Figure 2. Tumor size, GGO rate, and lymphatic involvement. Nine patients had lymphatic involvement, and 3 had both lymphatic and vessel involvement, while none had a lesion with only vessel involvement. GGO rate and tumor size were compared in the 9 patients with lymphatic involvement. One patient classified as Group II had a 7-mm solid component. *Two cases overlapped.

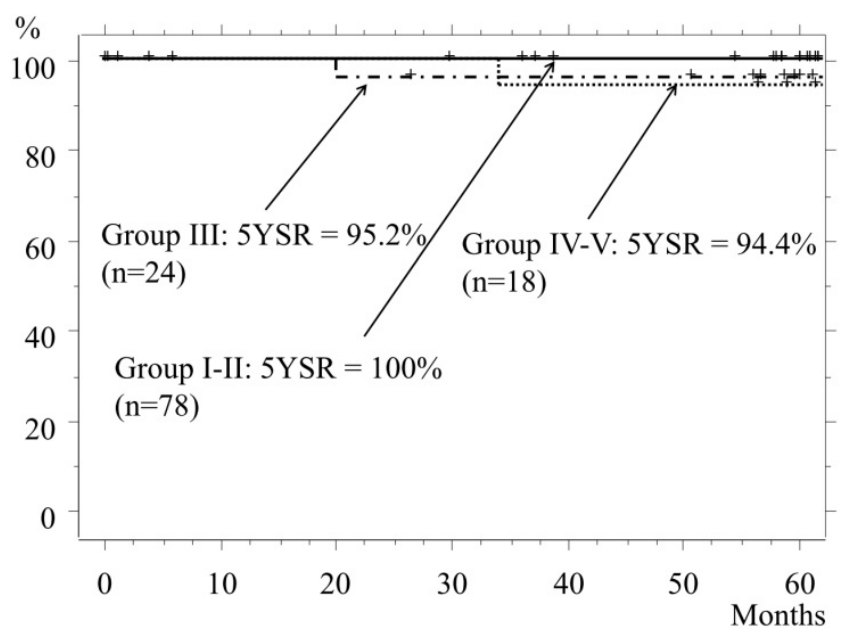

Figure 3. Overall survival. The 5YSR for Group I and II patients is $100 \%$, and most of those underwent a wedge resection or segmentectomy. The 5YSR for Group III and Group IV-V is $95.8 \%$ and $94.4 \%$, respectively (III vs. IV-V, $p=0.53$ ).

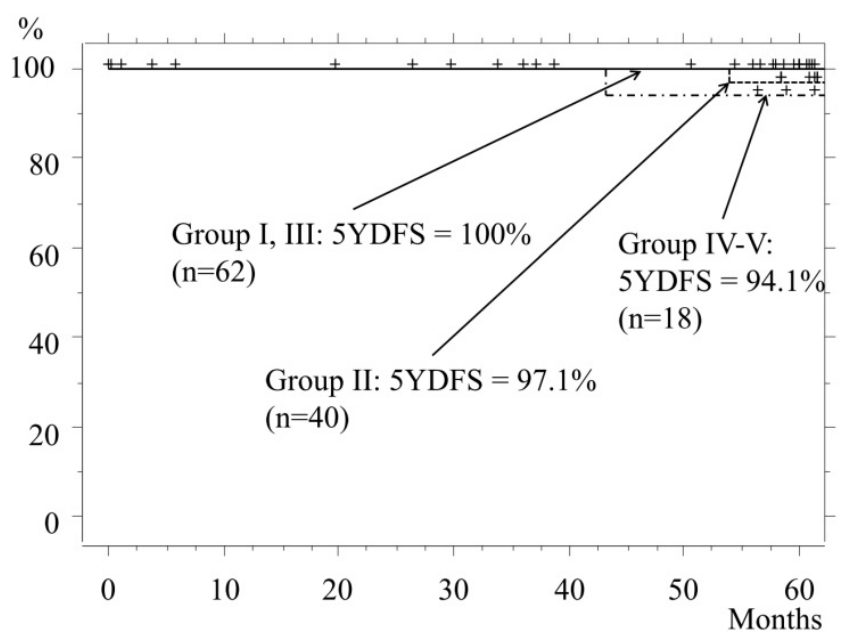

Figure 4. Disease-free survival. The 5YDFS rates for patients classified as Group I and Group II are $100 \%$ and $97.1 \%$, respectively, while those in Group III and Group IV-V are $100 \%$ and $94.1 \%$, respectively.

\section{Disease-free survival}

The 5-year disease-free survival (5YDFS) rate for the entire cohort was $98.1 \%$, whereas the rates for Groups I and II were $100 \%$ and $97.1 \%$, respectively. In Group II, recurrence from the margin after undergoing a wedge resection occurred in 1 patient, in whom the tumor had a GGO rate of $55 \%$ and was $11 \mathrm{~mm}$ in size. Even though margin cytology findings were negative, recurrence appeared at 54 months (4.5 years) after the operation, for which the patient underwent re-operation with a lobectomy. After that surgery, neither recurrence nor metastasis was seen for at least 4 years. The 5YDFS rates for Groups III and IV-V were $100 \%$ and $94.1 \%$, respectively (Fig. 4).

\section{Discussion}

Previous studies have reported that the malignancy of a small size pulmonary adenocarcinoma could be predicted using cytological diagnosis of imprint smears during a standard operation. 9,10 Furthermore, they concluded that such findings could provide important clinical information for planning the surgical procedure. However, there are no known reports of cytological results being applied to a surgical procedure. This is the first study of the clinical application of cytological classification in cases of small lung adenocarcinomas.

Several studies have presented the findings of limited resection for small pulmonary adenocarcinomas using intraoperative frozen sections. ${ }^{5,6}$ However, for tumor diagnosis with a frozen section, an adequately sized sample is required, which is difficult with small tumors. On the other hand, an imprint smear method only uses the surface of the section and can provide a diagnosis within 15 minutes. In addition, we previously reported that the N-H classification is correlated with the IASLC adenocarcinoma pattern classification, the $4^{\text {th }}$ WHO histological grading, and the $8^{\text {th }}$ IASLC/ATS/ERS classification. ${ }^{8}$ Therefore, for intraoperative small tumor diagnosis, a cytological approach has some advantages over a frozen section method.

The indications for surgery in the present patients were scrupulously selected. Tumors that were $\leq 2 \mathrm{~cm}$ in size or $>1.5 \mathrm{~cm}$ with less than $50 \%$ GGO were included. We previously reported that the GGO rate was related to prognosis in patients with small adenocarcinomas. ${ }^{11}$ In addition, another of our previous reports showed that the survival and disease-free rates of patients after a lobectomy for adenocarcinomas sized $16-20 \mathrm{~mm}$ and containing less than 50\% GGO were significantly worse than for patients who underwent wedge resection for GGO-predominant adenocarcinomas. ${ }^{12}$ 
Cases with mucinous lesions were not included in the present study, because the smear sample from them contains mainly mucus and histiocytes, rarely tumor cells. In addition, a mucinous adenocarcinoma can easily spread in an aerogenous manner. ${ }^{13}, 14$ Furthermore, their tumor cells can spread from a macroscopic lesion widely throughout the surrounding lung parenchyma in an area larger than expected. Therefore, we considered that limited surgery was not indicated for cases with a mucinous lesion.

For preoperative diagnosis, positron emission tomography (PET) scanning has been used to diagnose a tumor's malignancy. We began to use PET scans from 2003. In the beginning, we did the scans only for selected cases. The examination has now been routinely performed since 2010 . Therefore, PET scans were not available for all cases in the present cohort, but only for 57 cases. Just for information, the N-H classification correlated with the SUV-max of the PET scan $(R=0.577$, Supplement Figure 3$)$. However, a PET scan can be affected not only by the tumor's malignancy, but also by its size and tissue density. In small tumors, there can often be false-negative tumors based on the SUV-max value (as a reference, see also Supplement Figure 3). This means that, if SUV-max is high, a lobectomy (or standard operation) is needed, but if the SUV-max is low, we cannot say that the tumor is non-invasive carcinoma. A low SUV-max can include also, for example, minimally invasive adenocarcinoma. Unfortunately, to determine whether a wedge resection is indicated, diagnosis by PET scan cannot be considered sufficient.

The patient in Group II with positive lymphatic invasion had no recurrence 5 years after the limited operation, while others with positive lymphatic invasion underwent a lobectomy. Lymphatic and vessel involvement was largely avoided with the limited resection of our therapeutic strategy.

The outcomes of Group I-II were acceptable even after the limited resection procedure, with acceptable disease-free survival in the Group I patients. However, 1 patient classified as Group II had margin recurrence. A detailed retrospective examination of the sample used for margin cytology showed only a few small carcinoma cells on the slide. Therefore, the difference between Group I and Group II was not caused by a difference in malignancy, but rather a limitation of the margin scanning procedure. Fortunately, that patient underwent a re-operation 4.5 years after the first operation and has since shown a good outcome.

\section{Conclusion}

Use of cytological findings along with the $\mathrm{N}-\mathrm{H}$ classification for determining operation extent is effective for patients with small pulmonary adenocarcinomas.

\section{Limitations}

This study was conducted at a single institute, and the number of subjects, especially in Group V, was inadequate for definitive conclusions. In addition, the N-H classification is a qualitative system, though we are attempting to improve the scoring system for quantitation.

In our institute, we have used video-assisted thoracic surgery (VATS) since 2000. First, we used VATS technique only for selected cases with a small thoracotomy. Since 2010, we do complete VATS for patients with a small tumor. Because of the diverse indication for VATS and the gradual change of the procedure, it was not mentioned in this article.

Furthermore, the same samples were diagnosed and scored at multiple institutes, with the results compared.

\section{Abbreviations}

TN: Investigation, Data curation, Writing original draft; TN: Conceptualization; TT: Data curation; AT: Investigation, Resources; HK, HI: Data curation; YT, SN, HN: Investigation, Resources; JO: Data curation; MH: Conceptualization, Data curation, Formal analysis, Methodology, Project administration, Writing - review \& editing.

\section{Supplementary Material}

Supplementary figures and tables. http://www.jcancer.org/v11p2724s1.pdf

\section{Competing Interests}

The authors have declared that no competing interest exists.

\section{References}

1. Deschamps C, Pairolero PC, Trastek VF, Payne WS. Multiple primary lung cancers. Results of surgical treatment. J Thorac Cardiovasc Surg. 1990; 99: 769-77.

2. Ribet M, Dambron P. Multiple primary lung cancers. Eur J Cardiothorac Surg. 1995; 9: 231-6.

3. Van Meerbeeck J, Weyler J, Thibaut A, et al. Second primary lung cancer in Flanders: frequency, clinical presentation, treatment and prognosis. Lung Cancer. 1996; 15: 281-95.

4. Rice D, Kim HW, Sabichi A, et al. The risk of second primary tumors after resection of stage I nonsmall cell lung cancer. Ann Thorac Surg. 2003; 76: 1001-7.

5. Ichiki $Y$, Hanagiri $T$, Baba $T$, et al. Limited pulmonary resection for peripheral small-sized adenocarcinoma of the lung. Int J Surg. 2011; 9: 155-9.

6. Yoshida J, Nagai K, Yokose T, et al. Limited resection trial for pulmonary ground-glass opacity nodules: fifty-case experience. J Thorac Cardiovasc Surg. 2005; 129: 991-6.

7. Rami-Porta R, Bolejack V, Crowley J, et al. The IASLC Lung Cancer Staging Project: Proposals for the Revisions of the T Descriptors in the Forthcoming Eighth Edition of the TNM Classification for Lung Cancer. J Thorac Oncol. 2015 Jul;10(7):990-1003.

8. Nakagiri T, Tokunaga T, Nakayama T, et al. Novel Intraoperative Imprint Cytological Classification for Small Pulmonary Adenocarcinoma, Comparison with the $8^{\text {th }}$ Lung Cancer Stage and Adenocarcinoma Pattern Classification. J Cancer 2020. 
9. Kobayashi Y, Yokose T, Kawamura K, et al. Cytologic factors associated with prognosis in patients with peripheral adenocarcinoma of the lungmeasuring 3 $\mathrm{cm}$ or less in greatest dimension. Cancer. 2005; 105: 44-51.

10. Maezawa N, Tsuta K, Shibuki Y, et al. Cytopathologic factors can predict invasion in small-sized peripheral lung adenocarcinoma with a bronchioloalveolar carcinoma component. Cancer. 2006; 108: 488-93.

11. Kodama K, Higashiyama M, Takami K, et al. Treatment strategy for patients with small peripheral lung lesion(s): intermediate-term results of prospective study. Eur J Cardiothorac Surg. 2008; 34: 1068-74.

12. Higashiyama M, Oda $K$, Okami J, et al. Prognostic value of intraoperative pleural lavage cytology for lung cancer without carcinomatous pleuritis: importance in patients with early stage disease during long-term follow-up. Eur J Cardiothorac Surg. 2009; 35: 337-42.

13. Gaeta M, Blandino A, Pergolizzi $S$, et al. Patterns of recurrence of bronchioloalveolar cell carcinoma after surgical resection: a radiological, histological, and immunohistochemical study. Lung Cancer. 2003; 42: 319-26.

14. Travis WD, Brambilla E, Nicholson AG, et al. The 2015 World Health Organization Classification of Lung Tumors: Impact of Genetic, Clinical and Radiologic Advances Since the 2004 Classification. J Thorac Oncol. 2015 Sep;10(9):1243-1260. 